\title{
Treatment and management of advanced heart failure in elderly
}

\author{
Nidal Tourkmani ${ }^{1,2}$ \\ ${ }^{1}$ Co-Responsible of Preventive Cardiology and Rehabilitation Services, ABL, Swiss Prestige International Medical \\ Center, Guangzhou; ${ }^{2}$ Consultant Cardiologist, Guangdong General Hospital, Guangzhou, China
}

\begin{abstract}
Heart failure (HF) is a life-limiting condition, associated with high morbidity. End-stage, known as advanced heart failure (AHF), is more common among the elderly. HF patients' disease trajectory is more variable and unpredictable than the trajectory for most oncologic illnesses. Despite a growing armamentarium of resources, the management of AHF patients can be complex. Advances in medical therapy have dramatically improved the quality of life and survival of patients with end-stage HF. The majority of studies reveal lack of knowledge of HF among elderly patients. Mechanical circulatory support can provide bridgeto-transplantation therapy in eligible patients or destination therapy in those ineligible for heart transplantation like the majority of elderly patients with HF. The palliative care stage, considered as treatment basically aimed at controlling symptoms, may last a long time in some patients and should not simply be regarded as the final phase. Studies show that patients with AHF may have a poor understanding of their condition and its outcome and, therefore, guidelines recommend health care professionals to have an open communication with patients and their families about the AHF trajectory, including discussing their preferences for future care, acknowledging the risk of a sudden death, and the possibility of deactivation of devices (i.e., implantable defibrillators) in the end-of-life. This contribution is an attempt to have a brief overview of strategies for the management of HF terminal stage in elderly.
\end{abstract}

Correspondence: Nidal Tourkmani, Preventive Cardiology and Rehabilitation Services, ABL, Swiss Prestige International Medical Center, Guangzhou, China.

E-mail: nidaltou@gmail.com

Key words: Heart Failure, elderly, end-stage, palliative care, end-of-life.

Received for publication: 4 January 2019.

Accepted for publication: 9 Janaury 2019.

CC Copyright N. Tourkmani, 2019

Licensee PAGEPress, Italy

Monaldi Archives for Chest Disease 2019; 89:1032

doi: 10.4081/monaldi.2019.1032

This article is distributed under the terms of the Creative Commons Attribution Noncommercial License (by-nc 4.0) which permits any noncommercial use, distribution, and reproduction in any medium, provided the original author(s) and source are credited.

\section{Introduction}

In high-income countries, the mean age of patients referring to medical wards with symptoms of acute heart failure is almost 75 years, while octogenarians ( $\geq 80$ years) among them range between $21 \%$ and $38 \%$ [1-3]. Heart failure (HF) is currently the most common cause for hospitalization in the elderly and by 2030 it is expected that almost half of the medical resources for HF will be spent for the treatment of HF patients $>80$ years old age [1] and it stands to reason that the most terminal stage, known as advanced heart failure, is more common among the elderly [4]. Patients with AHF comprise an estimated $1 \%$ to $10 \%$ of the overall heart failure population $[5,6]$, and the increasing prevalence of HF in the elderly may be attributed to the outstanding improvements in the treatment of cardiovascular disease and the development of life-prolonging HF medication and device therapy. Moreover, HF with preserved ejection fraction (HFpEF) syndrome is being increasingly acknowledged as a different entity, affecting primarily the elderly population [1-7].

\section{Definition of advanced heart failure}

The Heart Failure Society of America defines the advanced heart failure (AHF) as 'those patients who have advanced, persistent HF with symptoms at rest despite repeated attempts to optimize pharmacologic and nonpharmacologic therapy' as shown by 1 or more of the following factors: frequent hospitalizations ( $\geq 3$ per year), chronic poor quality of life (QoL) with inability to accomplish activities of daily living, need for intermittent or continuous intravenous support, or consideration of assistive devices as destination therapy [8].

'Advanced', 'refractory', 'terminal' and 'end-stage' heart failure are interchangeable terms, all reflecting patients who should be evaluated for AHF therapies [5].

\section{Management strategies for patients with AHF}

Older patients are under-represented in clinical trials and evidence for an optimal treatment option for this special subgroup is lacking [9].

\section{Pharmacological therapy}

The typical presentation of AHF includes symptoms and signs of congestion associated with normal or elevated blood pressure. The presence of exertional dyspnoea, orthopnoea, paroxysmal noc- 
turnal dyspnoea, increasing body weight and peripheral oedema corroborate the diagnosis of AHF $[9,10]$. In addition, patients with advanced HF often experience pain, weakness, fatigue, nausea, anorexia, constipation, oedema, cough, altered mental status (confusion and delirium), anxiety, depression, and sleep disorders [11]. With increasing age, however, atypical clinical presentations become more common, and may delay a correct diagnosis. Indeed, many of the elderly may not have dyspnoea because of their sedentary lifestyle, and report only fatigue or exhibit an altered mental state [8].

The pharmacological treatment of HF has made extraordinary progress in the last thirty years thanks to the introduction of Angiotensin-converting enzyme inhibitors ACEIs/angiotensin receptor blockers ARBs, $\beta$-blockers $(\beta$-Bs) and Mineralocorticoid receptor antagonist (MRAs), which have drastically reduced HF mortality independently of the disease severity and still today represent the cornerstone of the therapy for this syndrome [12] (Table 1).

$\beta$-Bs are considered first-line therapy in the treatment of systolic HF. As the major randomized trials included a significant proportion of the elderly, the efficacy of $\beta$-Bs in the elderly is welldocumented. All elderly patients without a history of allergy or intolerance to (ACEIs) should be treated, starting with low doses. In contrast, (ARBs) should be considered only in patients who are intolerant to ACEIs due to cough, rash, or angioedema. Recently, the PARADIGM-HF trial demonstrated that a new class of pharmacological therapy, which combines the neprilysin inhibitor sacubitril with the ARB valsartan reduces cardiovascular mortality and hospitalization for HF as well as all-cause mortality compared with enalapril alone. The PARADIGM-HF enrolled a large proportion of patients aged $\geq 65$ years; efficacy and safety (hypotension, renal impairment, and hyperkalaemia) outcomes were similar across all age groups. Concerning the use of MRAs in the elderly, the RALES, the EPHESUS, and the EMPHASIS-HF trials showed a decreased mortality risk, regardless of age. Ivabradine can safely be prescribed in the elderly. The SHIFT trial demonstrated that, in HF patients with sinus rhythm, ivabradine reduces cardiovascular mortality and HF hospitalization in young as well as in elderly patients. Incidence of adverse events such as symptomatic bradycardia, asymptomatic bradycardia, and phosphenes similarly occurred in any of the age groups. The DIG trial has showed that digoxin reduces the risk of hospitalization with a higher risk of toxic effect and withdrawals in the elderly. In this regard, a serum digoxin concentration of $0.5-0.9 \mathrm{ng} / \mathrm{ml}$ is sufficient [13].

Currently, treatment of HF in elderly patients is characterized by widespread underutilization of the recommended therapies and suffers from the lack of clinical trials carried out on this particular segment of the population. Subgroups of the elderly population such as the over-80s are in fact totally devoid of specific therapeutic references, and a clinical picture such as HFpEF, which inevitably will be diagnosed more and more in the future, has not yet received adequate attention in the scientific literature [12].

\section{Short-term management}

AHF therapies refer to long-term mechanical circulatory support (MCS) or cardiac transplantation. However, in situations where the patient's clinical condition deteriorates, or end-organ function is compromised, short-term therapies may be needed until MCS can be implanted or while the patient is waiting on the transplant list [5].

\section{1) Intravenous vasoactive drugs}

It is well known that inotropes may improve haemodynamics and help reverse worsening end-organ function in AHF. Inotropic therapy may be used as a bridge strategy, but it is only a palliative measure when used on its own, because of the lack of outcomes data [5].

\section{2) Management of congestion}

Most of the hospitalizations are due to signs and symptoms of fluid overload. Recurrent congestion worsens patients' outcomes. Loop diuretics remain the cornerstone for the treatment of congestion in the patients with heart failure [5].

In AHF, medical treatment consists largely of the conventional medical classes, including diuretics, vasodilators, opioids and oxygen. Oxygen treatment appears to be superior to room air in relieving dyspnoea only in patients with hypoxia. In addition to smalldose opioids, benzodiazepines may reduce the anxiety associated with breathlessness. Pain is common, but is often undertreated in end-stage heart failure. Opioids are used as first-line treatment, starting with short-acting agents and then switching to sustainedrelease preparations when total daily requirements are determined. Oral or transdermal nitrates and other vasodilators are effective for treating anginal pain. Non-steroidal anti-inflammatory drugs should be avoided because of the risk of fluid retention, renal failure and gastrointestinal bleeding [9].

\section{3) Short-term mechanical circulatory support}

Among patients with AHF, short-term MCS [Intra-aortic balloon pump; Extracorporeal membrane oxygenation; TandemHeart ${ }^{\circledR}$ percutaneous ventricular assist device (Cardiac Assist, Inc., Pittsburgh,

\section{Table 1. Management of HF drugs therapy in elderly patient.}

1) $\beta$-Bs, ACEI / ARBs and MRAs have proven efficacy in improving symptoms and quality of life as well as reducing mortality. It is recommended to keep these drugs in the absence of side effects.

2) If symptomatic hypotension occurs try disconcerting doses of medications.

3) If this does not work the number of drugs the patient takes to start to find a combination of doses that the patient can tolerate begins to decrease.

4) There is not complete agreement on the order of medication reduction in this patient population; however, the following presents the general agreement. First: If the patient has hypotension, stop calcium channel blockers.

Second: If hypotension continues, consider stopping $\alpha$-blocking medications next.

Third: Try to maintain the $\beta$-blocker and the ACEI if possible; however, often the doses will need to be reduced.

Fourth: Whether or not to continue anticoagulation is often a concern. Currently, this decision is best made on an individual basis with each patient. Exploring the patient's values and goals, as well considering the burden associated with anticoagulation therapy, usually leads to a satisfactory decision. Fifth: Finally, review all your patient's medications, as there are often other medications that can be eliminated at this time, such as cholesterol-lowering agents [8]. 
PA, USA); Impella ${ }^{\circledR}$ ventricular support systems (Abiomed Inc., Danvers, MA, USA); CentriMag acute circulatory support system (St. Jude, Minneapolis, MN, USA); etc.] may be indicated in the setting of cardiogenic shock and can be used as a bridge-to-decision for long-term MCS or heart transplantation (HT). Several percutaneous and paracorporeal devices are available which can be used for a few days, up to several weeks, to allow cardiac recovery as well as recovery of other organs such as the kidneys, liver, and brain [5].

\section{Long-term management of AHF}

\section{Surgical options}

The decision to leverage these options must be balanced with not only age, but also frailty, comorbidities, and cognition, functional, social and nutritional status to determine the right candidates for each therapy [4]. Comorbidities can complicate the evaluation of patients with HF, and sometimes influence candidacy for MCS or HT, although it should be recognized that in some cases comorbidities may improve after application of advanced therapies. End-organ damage, in particular kidney or liver dysfunction and pulmonary hypertension, may be a consequence of acute congestion and/or lowoutput state, but it may be difficult to distinguish primary and secondary dysfunction or to predict reversibility [5].

The rational allocation of heart replacement options HT or Left Ventricular Assist Device Therapy (LVAD) among elderly patients requires an understanding of risk and benefit in the context of clinical care, disease trajectory and, particularly, noncardiac comorbidities (renal insufficiency, chronic lung disease, neuromuscular or cognitive disorders, nutrition and psychosocial isolation, etc.), including frailty [4].

\section{1) Heart Transplantation (HT) [4]}

Approximately $1 \%-3 \%$ of all transplants are still performed in patients at least 70 years old highly selected, and several case series have demonstrated similar intermediate outcomes (at 1 and 3 years) between older (age $>70$ ) and younger HT recipients. It had been accepted that advanced age adversely affects long-term survival rates in HT recipients.

\section{2) Left Ventricular Assist Device Therapy (LVAD) [4]}

In previous pivotal trials of LVAD technologies, the most common indication for destination therapy was age, and subsequent trials or registries have dramatically increased the use of LVAD among elderly patients.

There are an increasing number of elderly patients with AHF who are not candidates for HT or an LVAD or who do not wish to pursue either of these surgical options. The objective of these patients' care should focus on how to improve QoL through palliative care [4].

\section{Palliative care and end-of-life}

Since frailty appears to disproportionately affect the elderly and has a greater influence on quality and quantity of life, rational treatment options deliberated for elderly patients with AHF must include its consideration. Frail individuals have limited reserve for recovery from even transient major physiologic insults such as major surgery, prolonged intubation or intensive care time, hypoxia, hypotension or metabolic derangements [4].
In many cases, LVAD or HT is not medically or surgically viable for these patients, and some patients who understand their prognosis still choose not to pursue these options. At this point, the focus of treatment shifts from life-prolonging interventions to quality improvements of the remaining life and the goals-of-care become managing physical symptoms and the psychological wellbeing of both the patient and family.

Successful PC must involve shared care through a multidisciplinary approach. Patients and their caregivers should be able to easily communicate with primary care, specialist palliative care services and the specialized AHF service, according to the resources of each centre $[5,14,15]$ The PAL-HF (Palliative Care in Heart Failure) trial, a single-centre study of 150 patients, showed that interdisciplinary palliative care intervention in AHF patients resulted in greater benefits in QoL, anxiety, depression and spiritual wellbeing compared with usual care alone [4-14]. The SWAPHF (Social Worker-Aided Palliative Care Intervention in High-risk Patients with Heart Failure) trial showed that patients at high risk for mortality from HF frequently overestimate their life expectancy and a structured social worker-led palliative care (PC) intervention enhances prognostic understanding and patient-physician communication regarding goals of care $[5,17]$.

In heart failure, the trajectory of each patient is different. Some studies $[5,18]$ showed that the majority of patients with HF reject the idea of heart failure as a terminal disease and prefer to focus on day-to-day management and maintenance, despite obvious deterioration in disease stage and needs over time. A comprehensive end-of life (EoL) plan of care for each patient should be available. An important aspect is deciding when to discontinue advanced therapies [e.g., MCS, implantable cardiac defibrillator (ICD), or immunosuppressive treatment]. This decision should be taken by the patient whenever possible, or by the patient's caregiver, family, or hospital ethics committee if the patient is unable to independently convey their decisions. Support can be discontinued in the hospital, in hospice, or at home depending on patient and family preferences, feasibility, and local resources [5].

Whenever possible, collaborative models of care are important in the care of these patients. The collaborative team can include a primary care physician who is available for home-based care, an internist or cardiologist to support the primary care physician in decisions regarding HF therapy, and a PC physician to support the management of complex medication regimens in the patient's home $[8,19,20]$. Pathways of communication between caregivers and members of the team, which might include remote access for cardiology or PC support, should be established [8]. Because communication skills for addressing end-of-life issues and death are often not incorporated into medical training, clinicians need to be appropriately equipped to identify patients that might be candidates for advanced HF therapies and to recognize the optimal time for referral [11]. Physicians should be prepared to address the needs of patients who are clearly not eligible for AHF therapies, engage in discussions about changing goals of care, and optimize management strategies to lessen the symptomatic burden of AHF and improve QoL [5] and further define specific goals toward the EoL in the context of the patient's psychosocial support and spiritual belief systems. Treatment goals are often framed in the context of patient autonomy, dignity and choice of physical location (home or facility) [4].

Although it is a fact that mortality rates of end-stage HF patients are similar to those suffering from aggressive cancer, $\mathrm{HF}$ patients tend to overestimate their life expectancy [1]. For those, the trajectory is often stable for a period of time, and as their disease progresses, they experience repeated decompensations with 
return to near baseline function and health $[8,21]$. One of these challenges includes the trajectory of HF being much more variable and unpredictable than the trajectory for most oncologic illnesses. Models of PC, developed primarily for those with oncologic illness, need to be adapted to acknowledge these challenges, as an increasing number of patients being referred for palliative care have non-malignant illness such as HF. Educating patients about advanced HF and helping them understand their illness and illness trajectory can foster end-of-life discussions [8].

The European Society of Cardiology (ESC) and American College of Cardiology (ACC)/American Heart Association (AHA) recommendations propose this option for terminal stage HF patients nonetheless, the ethical and legal background is not entirely clear. $\mathrm{PC}$ is not an option reserved for patients facing imminent death, but should be also integrated in primary care of terminal HF patient [7].

\section{Implantable cardiac defibrillator deactivation}

Defibrillator deactivation is important when patients want to focus on comfort, as the defibrillator might provide electric shocks to the patient at the end of life, which can be distressing and uncomfortable. It is good to remember here that defibrillator function of any implantable device is independent from pacing or resynchronization therapy [8].

\section{Conclusions}

Heart failure in the elderly will continue to be an increasing health burden. Elderly patients have the right to adequate care and to be considered. They should not suffer unnecessarily, should be fully informed on their medical condition and must freely express their opinions on treatment strategies and/or possible interventions. While these represent the majority of the end-stage HF population and have a worse prognosis compared with the younger cohort, targeted treatment strategies have been insufficiently developed for them. Present knowledge is limited by low levels of enrolment of patients $\geq 70$ years old age in most trials. Future trials may address these issues by considering appropriate inclusion criteria and a thorough assessment of elderly patients. Finally, implementation strategies like palliative care and management of endof-life provide an additional clinical challenge, requiring a targeted and multidisciplinary approach and should be empirically investigated in this population.

\section{References}

1. Katsanos S, Bakosis G, Frogudaki A. Acute heart failure syndrome in the elderly. Continuing Cardiol Educ 2017;3:93-9.

2. Mizuno M, Kajimoto K, Sato N, et al. Clinical profile, management, and mortality in very-elderly patients hospitalized with acute decompensated heart failure: An analysis from the ATTEND registry. Eur J Intern Med 2016;27:80-5.

3. Ferrucci L, Giallauria F, Guralnik JM. Epidemiology of aging. Radiol Clin North Am 2008;46:643-52.

4. Guzman-Gutierrez G, Shi Y, Rappelt M, et al. Advanced heart failure treatment options among the elderly. Cent Res Rev 2016;3:199-206.
5. Crespo-Leiro MG, Metra M, Lund LH, et al. Advanced heart failure: a position statement of the Heart Failure Association of the European Society of Cardiology. Eur J Heart Fail 2018;20: 1505-35.

6. Bjork JB, Alton KK, Georgiopoulou VV, Butler J, Kalogeropoulos AP. Defining advanced heart failure: a systematic review of criteria used in clinical trials. J Card Fail 2016;22:569-77.

7. Andersson C, Vasan RS. Epidemiology of heart failure with preserved ejection fraction. Heart Fail Clin 2014; 10:377-88.

8. Steinberg L, White M, Arvanitis J, et al. Approach to advanced heart failure at the end of life. Can Fam Physician 2017;63: 674-80.

9. Teixeira A, Arrigo M, Tolppanen H, et al. Management of acute heart failure in elderly patients. Arch Cardiovasc Dis 2016;109:422-30.

10. Pedretti RFE, Fattirolli F, Griffo R, et al. Cardiac Prevention and Rehabilitation "3.0": From acute to chronic phase. Position Paper of the ltalian Association for Cardiovascular Prevention and Rehabilitation (GICR-IACPR). Monaldi Arch Chest Dis 2018;88:1004.

11. Whellan DJ, Goodlin SJ, Dickinson MG, et al. End-of-life care in patients with heart failure. J Card Fail 2014;20:121-34.

12. Scorcu G, Pilleri A. Treatment of heart failure in the elderly: Which drugs are essential and which should be avoided. Monaldi Arch Chest Dis 2018;88:948.

13. Guerra F, Brambatti M, Matassini MV, Capucci A. Current therapeutic options for heart failure in elderly patients. BioMed Res Int 2017;2017:1483873.

14. Ponikowski P, Voors AA, Anker SD, et al. 2016 ESC Guidelines for the diagnosis and treatment of acute and chronic heart failure: The Task Force for the diagnosis and treatment of acute and chronic heart failure of the European Society of Cardiology (ESC). Developed with the special contribution of the Heart Failure Association (HFA) of the ESC. Eur J Heart Fail 2016;18:891-975.

15. Feldman D, Pamboukian SV, Teuteberg JJ, et al. The 2013 International Society for Heart and Lung Transplantation Guidelines for mechanical circulatory support: executive summary. J Heart Lung Transplant 2013;32:157-87.

16. Rogers JG, Patel CB, Mentz RJ, et al. Palliative care in heart failure: the PAL-HF randomized, controlled clinical trial. J Am Coll Cardiol 2017;70:331-41.

17. O'Donnell AE, Schaefer KG, Stevenson LW, et al. Social worker-aided palliative care intervention in high-risk patients with heart failure (SWAP-HF): a pilot randomized clinical trial. JAMA Cardiol 2018;3:516-9.

18. Stocker R, Close H, Hancock H, et al. Should heart failure be regarded as a terminal illness requiring palliative care? A study of heart failure patients', carers' and clinicians' understanding of heart failure prognosis and its management. BMJ Support Palliat Care 2017;7:464-9.

19. Johnson M, Nunn A, Hawkes T, et al. Planning for end-of life care in heart failure: experience of two integrated cardiologypalliative care teams. Br J Cardiol 2012;19:71-5.

20. Allen LA, Stevenson LW, Grady KL, et al. Decision making in advanced heart failure: a scientific statement from the American Heart Association. Circulation 2012;125:1928-52.

21. McClung JA. End-of-life care in the treatment of advanced heart failure in the elderly. Cardiol Rev 2013;21:9-15. 